\title{
PACE4 regulates apoptosis in human pancreatic cancer Panc-1 cells via the mitochondrial signaling pathway
}

\author{
XIAO-FENG TIAN, GUO-MIN HUANG, HONG-LIANG ZANG and HONG CAO \\ Department of General Surgery, Xinmin Branch of The China-Japan Union Hospital, \\ Jilin University, Changchun, Jilin 130021, P.R. China
}

Received April 11, 2015; Accepted April 7, 2016

DOI: $10.3892 / \mathrm{mmr} .2016 .5885$

\begin{abstract}
Previous studies have demonstrated the overexpression of paired basic amino acid cleaving enzyme 4 (PACE4) mRNA in prostate cancer tissues. This overexpression is correlated with higher circulating protein levels in certain patients, however, the role of PACE4 in apoptosis and the potential molecular mechanisms of pancreatic cancer remain to be elucidated. The aim of the present study was to investigate the effect and potential molecular mechanisms of PACE4 on apoptosis in the Panc-1 pancreatic cancer cell line. Cell proliferation was assessed using a Cell Counting Kit-8 assay. Apoptotic nuclear shrinkage was monitored using Hoechst 33258 staining. Caspase-3/7 activities were measured using a colorimetric caspase-glo 3/7 assay. Alterations in protein expression were monitored using Western blot analysis. The results indicated that PACE4 small interfering (si)RNA inhibited cell proliferation and activated caspase-3/7 activities. In addition, PACE4 siRNA significantly increased apoptosis via the activation of caspase-3 and the downregulation of anti-apoptotic proteins, $\mathrm{X}$-linked inhibitor of apoptosis protein and phosphorylated-Akt. In addition, the results showed deregulation of the B cell lymphoma-2 (Bcl-2)-associated $\mathrm{X}$ protein/Bcl-2 ratio which led to the release of cytochrome $c$ following PACE4 siRNA transfection. In conclusion, PACE4 siRNA may exert antitumor activity through the mitochondrial pathway and is expected to be a promising therapeutic strategy for the treatment of pancreatic cancer.
\end{abstract}

\section{Introduction}

Pancreatic cancer, one of the most aggressive types of human malignancy, has a poor prognosis with a five-year survival

Correspondence to: Professor Hong Cao, Department of General Surgery, Xinmin Branch of The China-Japan Union Hospital, Jilin University, 829 Xinmin Road, Chaoyang, Changchun, Jilin 130021, P.R. China

E-mail: caohongcc@yeah.net

Key words: paired basic amino acid cleaving enzyme 4, pancreatic cancer, apoptosis, mitochondrial signaling rate of $<5 \%$ worldwide (1). It is characterized by rapid disease progression without specific symptoms, thus, early diagnosis and curative treatment are almost impossible $(1,2)$. The standard treatment strategies for pancreatic cancer include surgery, which is restricted to early disease stages, radiation and/or gemcitabine-based chemotherapies. However, pancreatic cancer is among the most intrinsically resistant types of malignancy to radiation and chemotherapy, resulting in an overall survival rate of $<7 \%$. Therefore, scientists and oncologists are attempting to identify novel and more efficient anti-pancreatic cancer agents or adjuvants (3-5). A previous report showed that $\sim 90 \%$ of patients succumb to the disease within 1 year following diagnosis, and the five-year survival rate is $<5 \%$ worldwide (6). The incidence of pancreatic cancer has been increasing in previous years, demonstrating the importance of investigating its pathogenesis. Alterations in gene and protein expression levels, and the activation of signaling pathways are associated with the occurrence and progression of pancreatic cancer (7).

Proprotein convertases (PCs) are a family of enzymes, which are responsible for the activation of numerous protein precursors. At present, nine PCs have been identified, namely, furin, paired basic amino acid cleaving enzyme 4 (PACE4), PC1/3, PC2, PC4, PC5/6, PC7, PCSK 9 and SKI-1/S1P (8). PACE4 is considered to be important in the development and progression of cancer. It activates several biologically relevant substrates; and a number have been shown to be significantly involvement in tissue homeostasis and cancer growth $(9,10)$. Among these are numerous metalloproteinases, growth factors, growth factor receptors and adhesion molecules, which are directly associated with tumor development (11-14). PACE4 is expressed at low levels in several mammalian tissues and has been demonstrated to be upregulated in certain tumor cell lines, including murine squamous cell carcinoma (15). In addition, mice overexpressing PACE4 have been found to exhibit tumors with increased growth rates (16).

Previously, two independent studies demonstrated the overexpression of PACE4 mRNA in prostate cancer tissues $(17,18)$. This overexpression was correlated with higher circulating protein levels in certain patients (18). However, the possible role of PACE4 in apoptosis and the potential molecular mechanisms of pancreatic cancer remain to be elucidated. In the present study, molecular silencing with small interfering (si)RNA was used to knock down endogenously expressed 
PACE4 in the Panc-1 cell line, following which cell proliferation and the apoptotic response was examined.

\section{Materials and methods}

Reagents and antibodies. The following polyclonal antibodies were purchased from Proteintech Group, Inc. (Wuhan, China): Rabbit anti-human cleaved caspase-3 (19677-1-AP; 1:3,000), rabbit B cell lymphoma (Bcl)-2 (12789-1-AP; 1:3,000), rabbit Bcl-2-associated X protein (Bax; 50599-2-Ig; 1:3,000), rabbit cytochtome $c$ oxidase subunit IV and X-linked inhibitor of apoptosis protein (XIAP; 10037-1-Ig; 1:2,000) antibodies, and the mouse anti-GAPDH (MM-0163-P; 1:1,000) antibody. Polyclonal rabbit anti-human PACE4 (AB151562; 1:3,000) and cytochrome $c$ (ab154476; 1:3,000)antibodies were purchased from Abcam (Cambridge, UK). Polyclonal rabbit anti-human phosphorylated-Akt (p-Akt; 13038; 1:2,000) and Akt $(4685 ; 1: 3,000)$ antibodies were purchased from Cell Signaling Technologies, Inc. (Danvers, MA, USA). Anti-rabbit (15134-1-AP) and anti-mouse (30000-0-AP) IgG-horseradish peroxidase antibodies were purchased from Proteintech Group, Inc. A Cell Counting Kit-8 (CCK-8) and Hoechst 33258 were purchased from Beyotime Institute of Biotechnology (Haimen, China). Other reagents were of analytical grade.

Cell culture and small interfering RNA transfection. Human pancreatic cancer Panc-1 cells were obtained from American Type Culture Collection (Manassas, VA, USA). The cells were routinely grown in Dulbecco's modified Eagle's medium (DMEM; GE Healthcare Life Sciences, Beijing, China) containing 10\% fetal bovine serum (FBS; GE Healthcare Life Sciences), $100 \mathrm{U} / \mathrm{ml}$ penicillin (Sigma-Aldrich, St. Louis, MO, USA) and $100 \mu \mathrm{g} / \mathrm{ml}$ streptomycin (Sigma-Aldrich) at $37^{\circ} \mathrm{C}$ in a humidified atmosphere of $5 \% \mathrm{CO}_{2}$. The cultures were replaced every 2-3 days and the cultures were divided into two at $80 \%$ confluence.

The Panc-1 cells were transfected with $100 \mathrm{nM}$ of PACE4 siRNA (Santa Cruz Biotechnology, Inc., Dallas, TX, USA; cat. no. sc-45482; Genbank ID for PACE4, NM_174936) or control siRNA (scrambled siRNA, a universal negative control; Sangon Biotech Co., Ltd., Shanghai, China) with GeneSilencer siRNA transfection reagent (Genlantis, San Diego, CA, USA) at $37^{\circ} \mathrm{C}$, according to the manufacturer's protocol. At $48 \mathrm{~h}$ post-transfection, the efficiency of siRNA-mediated PACE4 knockdown was determined using Western blot analysis.

Cell proliferation assay. The Panc-1 cells were plated in each well of a 96-well plate at a density of $5 \times 10^{3}$ cells/well in the culture medium (Thermo Fisher Scientific, Inc., Waltham, MA, USA) at $37^{\circ} \mathrm{C}$. Following $24 \mathrm{~h}$ of incubation, the cells were transfected with PACE4 siRNA or control siRNA, as described above, for $12,24,36$ and $48 \mathrm{~h}$, followed by the addition of $10 \mu \mathrm{l} \mathrm{CCK}-8$ solution at $37^{\circ} \mathrm{C}$. The cells were then incubated for $3 \mathrm{~h}$ at $37^{\circ} \mathrm{C}$. Absorbance was measured at $450 \mathrm{~nm}$ using a spectrophotometer (NanoDrop ND-1000; Thermo Fisher Scientific, Inc.).

Morphological analysis following Hoechst 33258 staining. The Panc- 1 cells were seeded in 24 -well plates $\left(6 \times 10^{4}\right.$ cells per well) overnight, and transfected with PACE4 siRNA or control siRNA for $48 \mathrm{~h}$. The cells were then fixed and stained with Hoechst 33258 (Invitrogen; Thermo Fisher Scientific, Inc.). The apoptotic cells were visualized under a fluorescence microscope (M165 FC; Leica Microsystems GmbH, Wetzlar, Germany).

Detection of caspase-3/7 protein activity. The activities of caspase-3/7 were measured using a colorimetric method, according to the manufacturer's protocol, using a Caspase-glo 3/7 Assay kit (G8093; Promega Corporation, Madison, WI, USA). Briefly, $2 \times 10^{4}$ Panc- 1 cells were seeded in 96-well plates and, after $24 \mathrm{~h}$, the cells were transfected with PACE4 siRNA or control siRNA for another $48 \mathrm{~h}$. Subsequently, the lysates (lysed using Sigma-Aldrich lysis buffer) of the Panc-1 cells were mixed with equilibrated caspase-glo $3 / 7$ reagents for $1 \mathrm{~h}$ at room temperature. Luminescence was measured using a GloMax 96 luminometer (Promega Corporation).

Preparation of mitochondria and cytosol. A mitochondria/cytosol kit (C3601; Beyotime Institute of Biotechnology, Beijing, China) was used to isolate the mitochondria and cytosol, according to the manufacture's protocol. Following transfection, as described above, the cells $\left(5 \times 10^{7}\right.$ cells) were collected by centrifugation at $600 \mathrm{x} \mathrm{g}$ for $5 \mathrm{~min}$ at $4^{\circ} \mathrm{C}$, washed twice with ice-cold phosphate-buffered saline and then resuspended in $500 \mu \mathrm{l}$ isolation buffer (Tiangen Biotech Co., Ltd., Beijing, China) containing protease inhibitors for $10 \mathrm{~min}$ on ice. The cells were then mechanically homogenized using a Dunce grinder (ATX810; Tiangen Biotech Co., Ltd.). The unbroken cells, debris and nuclei were discarded by centrifugation at $800 \mathrm{x} \mathrm{g}$ for $10 \mathrm{~min}$ at $4^{\circ} \mathrm{C}$. The supernatants were centrifuged at $12,000 \mathrm{x}$ g for $15 \mathrm{~min}$ at $4^{\circ} \mathrm{C}$. The supernatant of the cytosol was collected and the pellet fraction mitochondria was dissolved in $50 \mu \mathrm{l}$ lysis buffer (Sigma-Aldrich).

Western blot analysis. The Panc-1 cells were transfected, as described above, and were then lysed using radioimmunoprecipitation assay buffer (Sigma-Aldrich). A Bicinchoninic Acid Protein Assay kit (NEP045-2; Beijing Dingguo Changsheng Biotechnology Co., Ltd., Beijing, China) was used to measure the protein concentrations. The total protein $(0.2 \mu \mathrm{g})$ was loaded onto 12\% SDS-PAGE gels (Tiangen Biotech Co., Ltd.) and transferred onto polyvinylidene fluoride membranes (Sigma-Aldrich). The membranes were probed overnight at $4^{\circ} \mathrm{C}$ with the indicated primary antibodies in Tris-buffered saline with Tween-20 (Tiangen Biotech Co., Ltd.), containing $1 \%$ bovine serum albumin (w/v; Gibco; Thermo Fisher Scientific, Inc.). The blots were then incubated for $1 \mathrm{~h}$ with anti-rabbit or mouse secondary antibodies at $37^{\circ} \mathrm{C}$. The immune complexes were detected using an ECL Detection kit (32132; Thermo Fisher Scientific, Inc.) and quantified using a scanning densitometer (SD4; Tobias Associates, Inc., Ivyland, PA, USA) with molecular analysis software (Quantity One 1-D Analysis software; version 4.6.9; Bio-Rad Laboratories, Inc., Hercules, CA, USA). Quantification of band density was additionally performed using Quantity One 1-D Analysis software with normalization to the GAPDH signal. The expression levels of the proteins of interest in the 
various treatment groups were expressed relative to those under non-treated conditions.

Statistical analysis. All data are presented as the mean \pm standard deviation, and were analyzed using Student's $t$-test and one-way analysis of variance to determine the levels of significance. $\mathrm{P}<0.05$ was considered to indicate a statistically significant difference. Statistical analysis was performed using SPSS/Windows 11.0 software (SPSS Inc., Chicago, IL, USA).

\section{Results}

PACE4 reduces Panc-1 cell proliferation. Panc-1 cell proliferation was examined using a CCK-8 assay following transfection of the cells with PACE4 siRNA or control siRNA. As shown in Fig. 1, PACE4 siRNA inhibited cell proliferation, with a significant reduction observed $48 \mathrm{~h}$ post-transfection, compared with that in the control siRNA-treated group $(\mathrm{P}<0.05)$. Thus, these data indicated that PACE4 may affect cellular proliferation in Panc-1 cells.

PACE4 siRNA induces the apoptosis of Panc-1 cells. In the present study, the Panc-1 cells were transfected with PACE4 siRNA. The results of the Western blot analysis indicated that the protein expression of PACE4 was inhibited in the PACE4 siRNA-transfected group, compared with the control siRNA-transfected group (Fig. 2A and B). In order to evaluate whether the proliferation inhibition induced by PACE4 siRNA in the Panc-1 cells was associated with apoptosis, the present study examined the morphological changes in the cells using Hoechst 33258 staining. The Panc-1 cells were transfected with PACE4 siRNA for $48 \mathrm{~h}$, and the apoptotic morphological changes were observed and compared with the appearances in the control group. In the control siRNA group, the nuclei of the Panc-1 cells were round and homogeneously stained (Fig. 2C). However, the PACE4 siRNA-transfected cells exhibited evident apoptotic characteristics, including cell shrinkage and membrane integrity loss or deformation, nuclear fragmentation and chromatin compaction of a late apoptotic appearance. Together, these data indicated that PACE4 siRNA induced apoptosis in the Panc-1 cells.

PACE4 siRNA induces apoptosis via a caspase-dependent pathway. Caspase- 3 is a critical executioner of apoptosis, and its activation is essential for DNA fragmentation and a number of the typical biochemical and morphological changes observed in cells undergoing apoptosis. Therefore, to evaluate whether PACE4 siRNA-induced apoptosis is involved in the activation of caspase-3/7, the present study investigated the activities of caspase-3/7 through measuring the bioluminescence intensities. The activities of caspase-3/7 were significantly activated following PACE4 siRNA transfection (Fig. 3A).

To further assess the role of PACE4 in Panc-1 cell apoptosis, the present study evaluated the expression levels of apoptosis-associated proteins. These included pro-apoptotic cleaved caspase-3 (c-caspase-3), anti-apoptotic XIAP and p-Akt. The results of the Western blot analysis and subsequent statistical analysis indicated that PACE4 siRNA increased the levels of c-caspase-3 by $\sim 2.2$-fold $(\mathrm{P}<0.05)$. By contrast, the

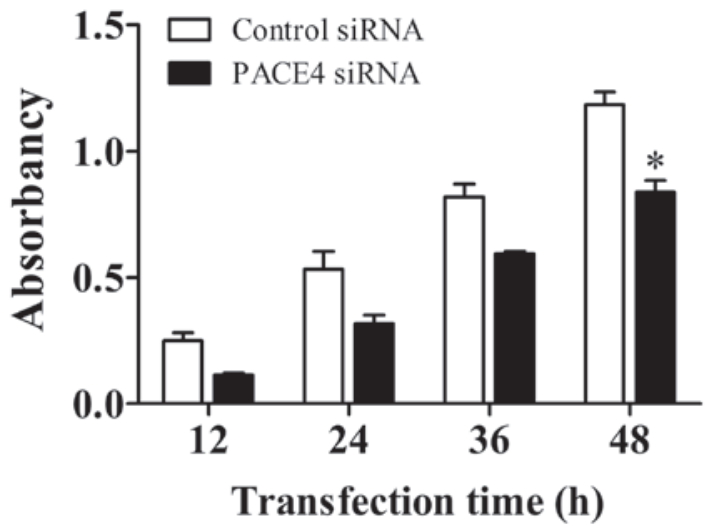

Figure 1. Proliferation of Panc-1 cells is inhibited by PACE4 siRNA. Panc-1 cells were transfected with PACE4 siRNA or control siRNA for the indicated durations. Cell proliferation was examined using a Cell Counting Kit- 8 assay. The OD value in each well was read at the wavelengths of 450 on a microtiter plate reader. Data are presented as the mean \pm standard deviation of OD450 at $12,24,36$ and $48 \mathrm{~h}$ of siRNA transfection. Each concentration was assessed in triplicate. ${ }^{*} \mathrm{P}<0.05$; PACE4 siRNA vs. control siRNA. PACE4, paired basic amino acid cleaving enzyme 4 ; siRNA, small interfering RNA; OD, optical density.

levels of XIAP and p-Akt were decreased by $~ 53 \%(\mathrm{P}<0.05)$ and $\sim 40 \%(\mathrm{P}<0.05)$, respectively, following PACE4 siRNA transfection (Fig. 3B and C).

PACE4 siRNA induces apoptosis via the mitochondrial signaling pathway. In order to further understand the molecular mechanisms by which PACE4 siRNA exerts pro-apoptotic effects, the present study examined the protein expression of mediators in the mitochondrial signaling pathway. Initially, the present study determined whether PACE4 siRNA stimulated the release of cytochrome $c$ into the cytosolic fraction in Panc-1 cells. As expected, cytochrome $c$ was redistributed following PACE4 siRNA transfection. The level of cytochrome $c$ in the mitochondria was significantly decreased by $64 \%$ (Fig. $4 \mathrm{~A}$ and $\mathrm{B} ; \mathrm{P}<0.05$ ). Correspondingly, the levels of cytochrome $c$ in the cytosol were increased by 1.83 -fold (Fig. 4C and D; $\mathrm{P}<0.05$ ).

As the Bcl-2 family of proteins are critical in regulating the release of cytochrome $c$, the present study subsequently investigated the possible involvement of Bax and Bcl-2 in the process of PACE4 siRNA-mediated Panc-1 cell apoptosis. As shown in Fig. 4E and F, the level of Bax was significantly increased and the level of Bcl-2 was markedly decreased in the PACE4 siRNA-transfected cells, compared with the control cells. Statistical analysis showed that PACE4 siRNA increased the ratio of $\mathrm{Bax} / \mathrm{Bcl}-2$ by $\sim 5.5$-fold $(\mathrm{P}<0.05)$.

\section{Discussion}

PACE4 has already been highlighted for its potential role in several types of neoplasia, including oral tongue carcinoma (19), hepatocellular carcinoma (20), glioma (21), skin cancer $(16,22)$ and prostate cancer $(17)$. Whereas previous studies have predominantly examined PACE4 overexpression, the present study focused on gene silencing as a predictive approach to define potential therapeutic benefits. In the present study, the effect of PACE4 siRNA on apoptosis in a cellular 
A

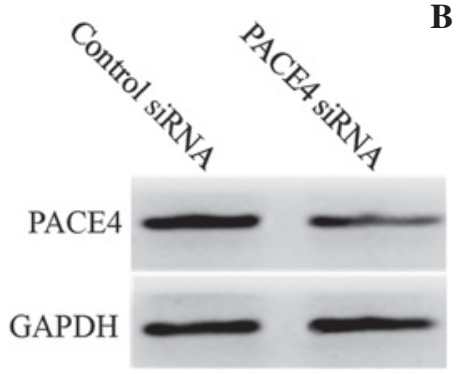

B

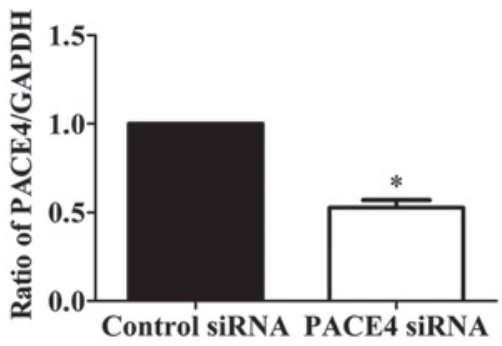

C

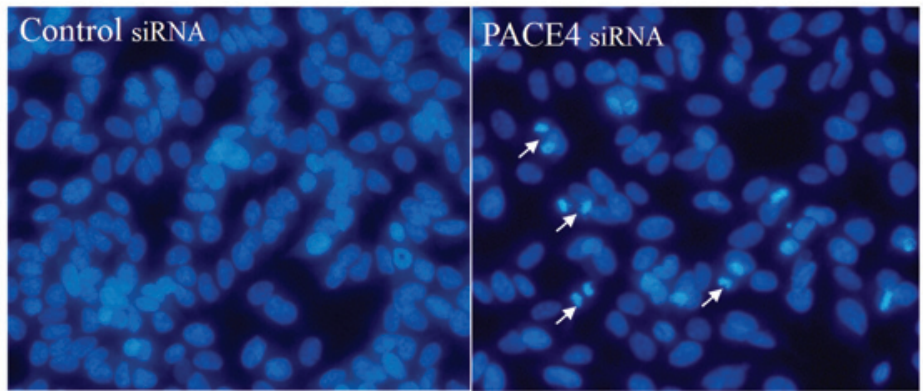

Figure 2. PACE4 siRNA significantly increases apoptosis of Panc-1 cells. Panc-1 cells were transfected with PACE4 siRNA or control siRNA for 48 h. (A and B) Expression of PACE4 in the Panc-1 cells following siRNA transfection. GAPDH was included as a loading control ("P<0.05, vs. control). The data are presented as the mean \pm standard deviation $(\mathrm{n}=3)$. (C) At $48 \mathrm{~h}$ post-transfection, the cells were incubated with Hoechst 33258 staining buffer. Healthy cells appeared round with intact nuclei, whereas apoptotic cells exhibited nuclear karyopyknosis or fragmentation, as indicated by the white arrows. Magnification, x100. PACE4, paired basic amino acid cleaving enzyme 4; siRNA, small interfering RNA.

A

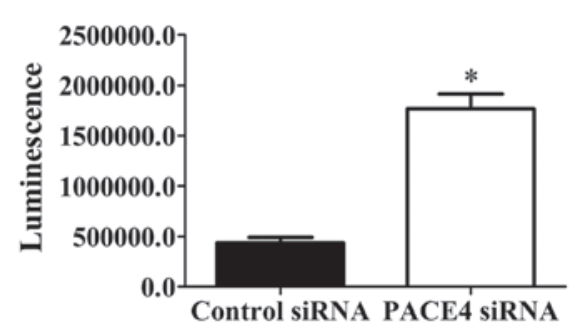

C

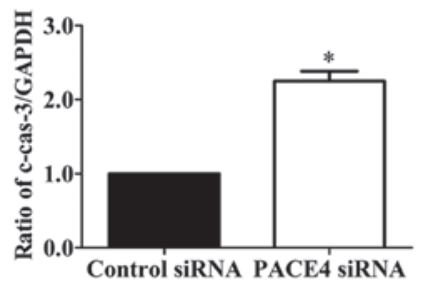

B
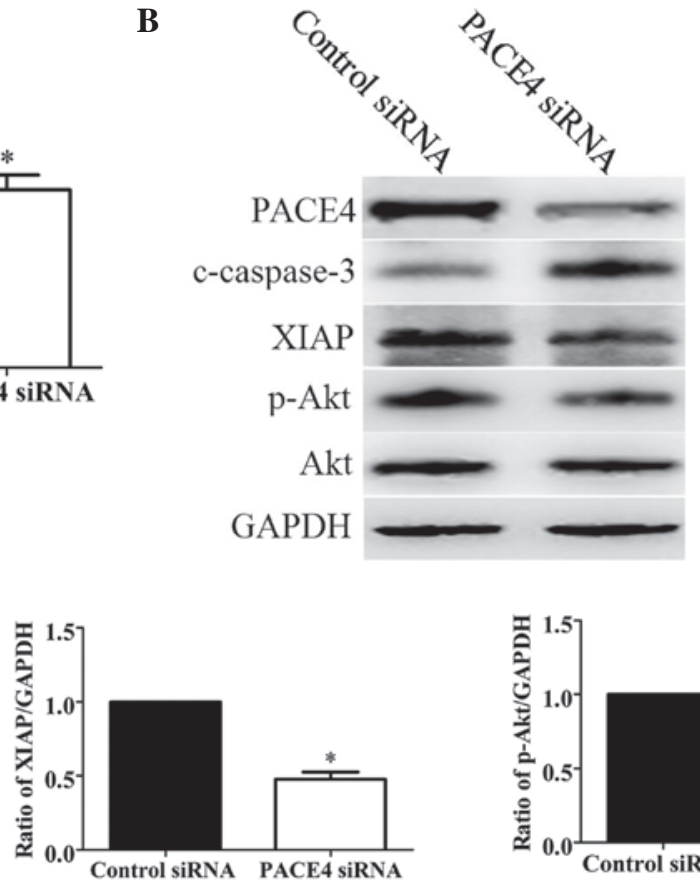

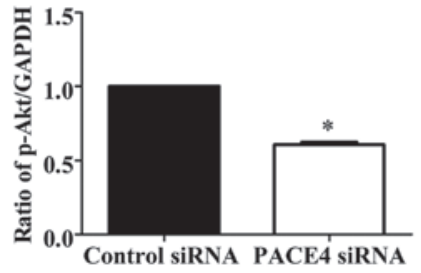

Figure 3. Western blot analysis of apoptotic-associated proteins following PACE4 siRNA transfection. (A) Relative luminescence expression levels of caspase-3/7 in Panc-1 cells transfected with PACE4 siRNA or control siRNA for $48 \mathrm{~h}$. The data are presented as the mean \pm standard deviation of three independent experiments. "P $<0.05$, compared with the control group. (B) Representative Western blot images. (C) Statistical analyses of the protein expression of c-cas, XIAP and p-Akt. Expression levels in three experiments were quantified by measuring the intensity of the apoptotic-associated proteins relative to GAPDH (loading control). The data are presented as the mean \pm standard deviation ( $\mathrm{n}=3$ ). ${ }^{*} \mathrm{P}<0.05$, vs. control. PACE4, paired basic amino acid cleaving enzyme 4; siRNA, small interfering RNA; c-cas-3, cleaved caspase 3; XIAP, X-linked inhibitor of apoptosis protein; p-Akt, phosphorylated Akt.

model of pancreatic cancer was investigated. The results indicated that PACE4 siRNA inhibited the proliferation of Panc-1 cells. Based on the results of Hoechst 33258 staining, measurement of caspase-3/7 activities and Western blot analysis, it was concludes that PACE4 siRNA induced apoptosis in the Panc-1 cells. Thus, the present study provided the first 
A

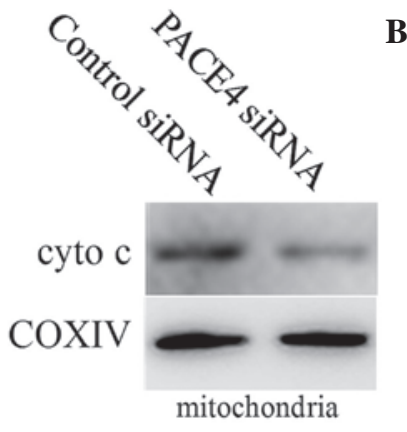

C

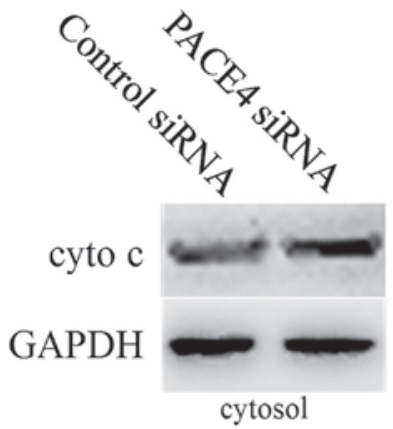

$\mathbf{E}$

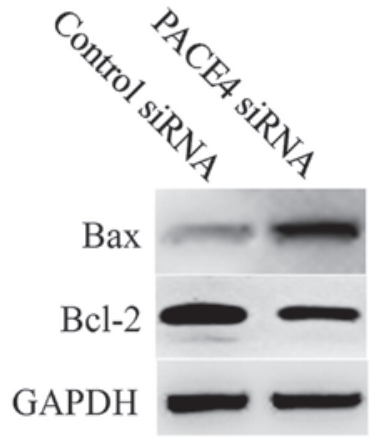

F
B

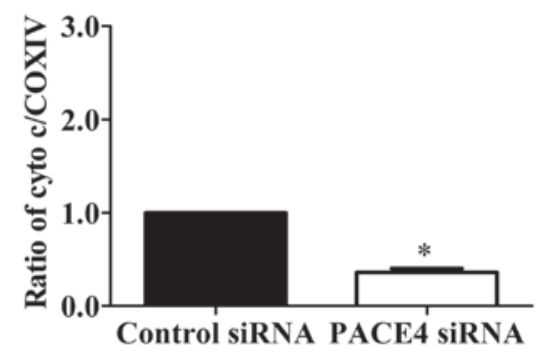

D

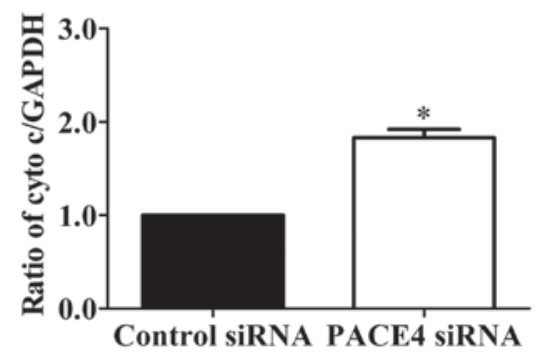

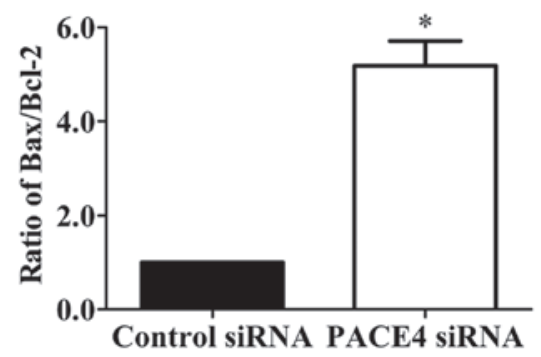

Figure 4. Regulation of mediators in the mitochondrial pathway in apoptotic Panc-1 cells by PACE4 siRNA. Panc-1 cells were transfected with PACE4 siRNA or control siRNA for $48 \mathrm{~h}$, and the levels of (A and B) cyto $\mathrm{c}$ in the mitochondria (COXIV as loading control) and (C and D) cytosol (GAPDH as a loading control) were measured using Western blot analysis. (E and F) PACE4 siRNA inhibited the expression of Bcl-2 and increased the expression of Bax, as detected by Western blot analysis. Intensities of the proteins of interest were measured for quantitative analysis. GAPDH was included as a loading control. The data are presented as the mean \pm standard deviation $(n=3)$. ${ }^{*} \mathrm{P}<0.05$, vs. control. PACE4, paired basic amino acid cleaving enzyme 4; siRNA, small interfering RNA; COXIV, cytochtome $c$ oxidase subunit IV; cyto c, cytochrome $c$; Bcl-2, B cell lymphoma-2; Bax, Bcl-2-associated X protein.

evidence, to the best of our knowledge, that PACE4 has an anti-apoptotic effect in pancreatic cancer cells.

As a primary executioner caspase in the majority of pathways of the caspase protein family, the activation of caspase-3 often results in the irreversible commitment of a cell to apoptosis. Therefore, the activation of caspase-3 is considered a reliable marker for cells undergoing apoptosis (23). The present study found that the activity of caspase-3/7 was significantly activated following PACE4 siRNA transfection (Fig. 3A). An effective strategy for destroying cancer cells is to induce cell apoptosis. XIAP, a member of the inhibitor of apoptosis protein family, contributes to the apoptosis resistance of cancer cells $(24,25)$. Akt is a promoter of cell proliferation and survival, and has been found to be overexpressed in tumor formation (26). Thus, the present study investigated whether these apoptosis-associated proteins were involved in PACE4 siRNA-induced apoptosis. The results of this investigation confirmed the role of PACE4 in the apoptosis of Panc-1 cells, based on the following lines of evidence: PACE4 siRNA increased the apoptosis of cells by regulating the expression levels of the apoptosis-associated factors c-caspase-3, XIAP and p-Akt (Fig. 3B and C). The inactivation of XIAP and p-Akt by PACE4 siRNA may prevent the development and progression of cancer.

The Bcl-2 family of proteins are important in the apoptosis of cancer cell apoptosis $(27,28)$. The Bcl-2 family can primarily regulate mitochondrial membrane permeabilization (29). The Bax/Bcl-2 ratio is usually regarded as a criterion for apoptosis (30). The results from the present study demonstrated that the level of cytochrome $c$ in the mitochondria was significantly decreased (Fig. 4A and B), whereas that in cytosol was increased (Fig. 4C and D). In addition, PACE4 siRNA increased the levels of Bax and decreased the level of $\mathrm{Bcl}-2$, leading to changes in the ratio of Bax/Bcl-2 (Fig. 4E and F). These results indicated that PACE4 siRNA had an effect on mitochondrial membrane stability. This was evidenced by the increased $\mathrm{Bax} / \mathrm{Bcl}-2$ ratio and the release of cytochrome $c$ into the cytoplasm. Taken together, these data demonstrated 
that PACE4 siRNA may exert its anti-tumor activity through the mitochondrial signaling pathway (intrinsic pathway) in pancreatic cancer cells.

In conclusion, the results of the present study suggested PACE4 siRNA possesses anti-proliferation and apoptosis-inducing properties in human pancreatic cancer Panc-1 cells. The PACE4 siRNA-induced apoptosis of Panc-1 cells may be mediated through the mitochondria pathway. These results support the potential of PACE4 to be developed as a promising agent for the treatment of pancreatic cancer.

\section{References}

1. Feng J, Ma T, Ge Z, Lin J, Ding W, Chen H, Zhu W, Zhou S and Tan Y: PKM2 gene regulates the behavior of pancreatic cancer cells via mitogen-activated protein kinase pathways. Mol Med Rep 11: 2111-2117, 2015.

2. Siegel R, Naishadham D and Jemal A: Cancer statistics, 2012. CA Cancer J Clin 62: 10-29, 2012.

3. Kim J, Kim YC, Fang C, Russell RC, Kim JH, Fan W, Liu R, Zhong Q and Guan KL: Differential regulation of distinct Vps34 complexes by AMPK in nutrient stress and autophagy. Cell 152: 290-303, 2013.

4. Gong L, Yang B, Xu M, Cheng B, Tang X, Zheng P, Jing Y and Wu GJ: Bortezomib-induced apoptosis in cultured pancreatic cancer cells is associated with ceramide production. Cancer Chemother Pharmacol 73: 69-77, 2014.

5. Min H, Xu M, Chen ZR, Zhou JD, Huang M, Zheng K and Zou XP: Bortezomib induces protective autophagy through AMP-activated protein kinase activation in cultured pancreatic and colorectal cancer cells. Cancer Chemother Pharmacol 74: 167-176, 2014.

6. Jin C, Yao L, Long J, Fu DL, Yu XJ, Yang F, Ni QX and $\mathrm{Xu} \mathrm{J}$ : Effect of multiple-phase regional intra-arterial infusion chemotherapy on patients with resectable pancreatic head adenocarcinoma. Chin Med J (Engl) 122: 284-290, 2009.

7. Preis M and Korc M: Signaling pathways in pancreatic cancer. Crit Rev Eukaryot Gene Expr 21: 115-129, 2011.

8. Seidah NG and Prat A: The biology and therapeutic targeting of the proprotein convertases. Nat Rev Drug Discov 11: 367-383, 2012.

9. Tsuji A, Sakurai K, Kiyokage E, Yamazaki T, Koide S, Toida K, Ishimura K and Matsuda Y: Secretory proprotein convertases PACE4 and PC6A are heparin-binding proteins which are localized in the extracellular matrix. Potential role of PACE4 in the activation of proproteins in the extracellular matrix. Biochim Biophys Acta 1645: 95-104, 2003.

10. Yuasa K, Masuda T, Yoshikawa C, Nagahama M, Matsuda Y and Tsuji A: Subtilisin-like proprotein convertase PACE4 is required for skeletal muscle differentiation. J Biochem 146: 407-415, 2009

11. Sato H, Kinoshita T, Takino T, Nakayama K and Seiki M: Activation of a recombinant membrane type 1-matrix metalloproteinase (MT1-MMP) by furin and its interaction with tissue inhibitor of metalloproteinases (TIMP)-2. FEBS Lett 393: 101-104, 1996.

12. Yana I and Weiss SJ: Regulation of membrane type-1 matrix metalloproteinase activation by proprotein convertases. Mol Biol Cell 11: 2387-2401, 2000.

13. Dubois CM, Blanchette F, Laprise MH, Leduc R, Grondin F and Seidah NG: Evidence that furin is an authentic transforming growth factor-beta1-converting enzyme. Am J Pathol 158: 305-316, 2001.
14. Khatib AM, Siegfried G, Prat A, Luis J, Chrétien M, Metrakos P and Seidah NG: Inhibition of proprotein convertases is associated with loss of growth and tumorigenicity of HT-29 human colon carcinoma cells: Importance of insulin-like growth factor-1 (IGF-1) receptor processing in IGF-1-mediated functions. J Biol Chem 276: 30686-30693, 2001.

15. Hubbard FC, Goodrow TL, Liu SC, Brilliant MH, Basset P, Mains RE and Klein-Szanto AJ: Expression of PACE4 in chemically induced carcinomas is associated with spindle cell tumor conversion and increased invasive ability. Cancer Res 57: 5226-5231, 1997.

16. Mahloogi H, Bassi DE and Klein-Szanto AJ: Malignant conversion of non-tumorigenic murine skin keratinocytes overexpressing PACE4. Carcinogenesis 23: 565-572, 2002.

17. D'Anjou F, Routhier S, Perreault JP, Latil A, Bonnel D, Foumier I, Salzet M and Day R: Molecular validation of PACE4 as a target in prostate cancer. Transl Oncol 4: 157-172, 2011.

18. Klee EW, Bondar OP, Goodmanson MK, Dyer RB, Erdogan S, Bergstralh EJ, Bergen HR III, Sebo TJ and Klee GG: Candidate serum biomarkers for prostate adenocarcinoma identified by mRNA differences in prostate tissue and verified with protein measurements in tissue and blood. Clin Chem 58: 599-609, 2012.

19. Estilo CL, O-charoenrat P, Talbot S, Socci ND, Carlson DL, Ghossein R, Williams T, Yonekawa Y, Ramanathan Y, Boyle JO, et al: Oral tongue cancer gene expression profiling: Identification of novel potential prognosticators by oligonucleotide microarray analysis. BMC Cancer 9: 11, 2009.

20. Kurokawa Y, Matoba R, Nakamori S, Takemasa I, Nagano H, Dono K, Umeshita K, Sakon M, Monden M and Kato K: PCR-array gene expression profiling of hepatocellular carcinoma. J Exp Clin Cancer Res 23: 135-141, 2004.

21. Delic S, Lottmann N, Jetschke K, Reifenberger G and Riemenschneider MJ: Identification and functional validation of CDH11, PCSK6 and SH3GL3 as novel glioma invasion-associated candidate genes. Neuropathol Appl Neurobiol 38: 201-212, 2012.

22. Bassi DE, Lopez De Cicco R, Cenna J, Litwin S, Cukierman E and Klein-Szanto AJ: PACE4 expression in mouse basal keratinocytes results in basement membrane disruption and acceleration of tumor progression. Cancer Res 65: 7310-7319, 2005.

23. Tait SW and Green DR: Mitochondria and cell death: Outer membrane permeabilization and beyond. Nat Rev Mol Cell Biol 11: 621-632, 2010.

24. LaCasse EC, Mahoney DJ, Cheung HH, Plenchette S, Baird S and Korneluk RG: IAP-targeted therapies for cancer. Oncogene 27: 6252-6275, 2008

25. Boonyarat $\mathrm{C}$, Yenjai $\mathrm{C}$, Vajragupta $\mathrm{O}$ and Waiwut $\mathrm{P}$ : Heptaphylline induces apoptosis in human colon adenocarcinoma cells through bid and Akt/NF-kB (p65) pathways. Asian Pac J Cancer Prev 15: 10483-10487, 2014.

26. Serrano ML, Sánchez-Gómez M, Bravo MM, Yakar S and LeRoith D: Differential expression of IGF-I and insulin receptor isoforms in HPV positive and negative human cervical cancer cell lines. Horm Metab Res 40: 661-667, 2008.

27. Cotter TG: Apoptosis and cancer: The genesis of a research field. Nat Rev Cancer 9: 501-507, 2009.

28. Leber B, Lin J and Andrews DW: Still embedded together binding to membranes regulates $\mathrm{Bcl}-2$ protein interactions. Oncogene 29: 5221-5230, 2010.

29. Adams JM and Cory S: The Bcl-2 apoptotic switch in cancer development and therapy. Oncogene 26: 1324-1337, 2007.

30. Jiang H, Zhao PJ, Su D, Feng J and Ma SL: Paris saponin I induces apoptosis via increasing the $\mathrm{Bax} / \mathrm{Bcl}-2$ ratio and caspase-3 expression in gefitinib-resistant non-small cell lung cancer in vitro and in vivo. Mol Med Rep 9: 2265-2272, 2014. 